\title{
Synthesis of Highly Functionalized Dihydropyridone and Pyridone Derivatives in Green Solvents
}

\author{
MARZIEH KHAZAEI \\ Young Researchers and Elite Club, Qom Branch, Islamic Azad University, Qom, Iran \\ miss.khazaei@gmail.com
}

Received 8 June 2014 / Accepted 29 June 2014

\begin{abstract}
A new and efficient one-pot synthesis of dihydropyridone and pyridone derivatives by four-component reaction between cyanoacetamide, aryl aldehydes and ethyl acetoacetate with ammonium in green solvents is described. The reaction was performed in water solvent under reflux conditions and afforded good yields of products.
\end{abstract}

Keywords: Dihydropyridone derivatives, Pyridone derivatives, Green solvents

\section{Introduction}

Substituted dihydropyridone and pyridone derivatives are important intermediates in the pharmaceutical, dye, and photo industries. ${ }^{1}$ pyridones are of interest because of the occurrence of their saturated and partially saturated derivatives in biologically active compounds and natural products such as NAD nucleotides, pyridoxol (vitamin $\mathrm{B}_{6}$ ), and pyridine alkaloids ${ }^{2}$. Due to their $\pi$-stacking ability, some pyridines are used in supramolecular chemistry ${ }^{3}$. Some examples are used as pharmaceuticals (as antimalarial, vasodilator, anesthetic, anticonvulsant, and antiepileptic), dyes, additives (as antioxidant), agrochemicals (as fungicidal, pesticidal, and herbicidal), veterinary (as anthelmintic, antibacterial, and antiparasitic), and also in qualitative and quantitative analysis ${ }^{4-7}$. So far, the most common synthetic methods for the preparation of pyridine ring systems involve: transformation of another ring and cyclizations classified on the basis of the number of ring atoms in each of the components being cyclized: from six ring atoms by $N-C_{\alpha}, C_{\alpha}-C_{\beta}$, or $C_{\beta}-C_{\gamma}$ bond formation; by formation of two bonds, from [5+1], [4+2], or [3+3] atom fragments; by formation of three bonds, from $[4+1+1],[3+2+1]$, or $[2+2+2]$ atom fragments; and by formation of four bonds, from $[3+1+1+1]$ or $[2+2+1+1]$ atom fragments ${ }^{8,9}$. Synthesis of 4-aryl-3-cyano-2,5-dihydro pyridin-2-one derivatives under solvent-free ${ }^{10}$ and ethanol ${ }^{11,12}$ conditions we reported.

\section{Experimental}

Melting points were determined with an Electrothermal 9100 apparatus. Elemental analyses were performed using a Costech ECS 4010 CHNS-O analyzer at analytical laboratory of 
Islamic Azad University, Yazd branch, Iran. Mass spectra were recorded on a FINNIGANMAT 8430 mass spectrometer operating at an ionization potential of $70 \mathrm{eV}$. IR spectra were recorded on a Shimadzu IR-470 spectrometer. ${ }^{1} \mathrm{H}$, and ${ }^{13} \mathrm{C}$ NMR spectra were recorded on BRUKER DRX-500 AVANCE spectrometer at 500.1 and $125.8 \mathrm{MHz}$, respectively. ${ }^{1} \mathrm{H}$, and ${ }^{13} \mathrm{C}$ NMR spectra were obtained on solution in $\mathrm{CDCl}_{3}$ using TMS as internal standard. Column chromatography was performed with Merck silica gel 60, 230-400 mesh. The chemicals used in this work were purchased from Fluka (Buchs, Switzerland) and were used without further purification.

\section{General procedure}

In a typical experiment, a mixture of pyridine $(10 \mathrm{~mol} \%)$, aryl aldehydes 1 ( $1 \mathrm{mmol})$ and cyanoacetamide $2(1 \mathrm{mmol})$ in water $(15 \mathrm{~mL})$ was stirred at room temperature for $24 \mathrm{~h}$ and was added to it a solution of ethyl acetoacetate $(1 \mathrm{mmol}) 3$ and $\mathrm{NH}_{4} \mathrm{OAc}(1 \mathrm{mmol})$ and was refluxed for $8 \mathrm{~h}$. After completion of the reaction, as indicated by TLC, the product was extracted with ethyl acetate $(10 \mathrm{~mL})$. The combined organic extracts were concentrated in vacuum and the resulting product was purified by column chromatography on silica gel with ethyl acetate and $n$-hexane $(1: 1)$ as eluent to afford the pure product.

\section{Ethyl 5-cyano-2-methyl-6-oxo-4-phenyl-1,4,5,6-tetrahydropyridine 3-carboxylate (1A)}

White powder, $(59 \%)$, m.p. $143-145^{\circ} \mathrm{C}$, IR $(\mathrm{KBr})\left(v_{\max } / \mathrm{cm}^{-1}\right): 3411(\mathrm{NH}), 2234(\mathrm{CN}), 1709$ $(\mathrm{C}=\mathrm{O})$. MS, m/z (\%): $284\left(\mathrm{M}^{+}\right.$, 5). Anal. Calcd for $\mathrm{C}_{16} \mathrm{H}_{16} \mathrm{~N}_{2} \mathrm{O}_{3}: \mathrm{C}, 67.59 ; \mathrm{H}, 5.67 ; \mathrm{N}, 9.85$ \%. Found: C, 67.43; H, 5.72; N, 9.75\%. ${ }^{1} \mathrm{H}$ NMR $\left(500.1 \mathrm{MH}_{\mathrm{Z}}, \mathrm{CDCl}_{3}\right): \delta=0.80\left(3 \mathrm{H}, \mathrm{t},{ }^{3} \mathrm{~J}_{\mathrm{HH}}\right.$ $\left.=7 \mathrm{H}_{\mathrm{Z}}, \mathrm{CH}_{3}\right), 2.38\left(3 \mathrm{H}, \mathrm{s}, \mathrm{CH}_{3}\right), 4.09\left(2 \mathrm{H}, \mathrm{q},{ }^{3} J_{\mathrm{HH}}=7 \mathrm{H}_{\mathrm{Z}}, \mathrm{CH}_{2}\right), 4.16\left(\mathrm{H}, \mathrm{d},{ }^{3} J_{\mathrm{HH}}=7.1 \mathrm{H}_{\mathrm{Z}}\right.$, $\mathrm{CH}), 4.47\left(\mathrm{H}, \mathrm{d},{ }^{3} \mathrm{~J}_{\mathrm{HH}}=7.1 \mathrm{H}_{\mathrm{Z}}, \mathrm{CH}\right), 7.26-7.61(5 \mathrm{H}, \mathrm{m}$, aromatic $), 13.38(1 \mathrm{H}$, broad s, NH$)$. ${ }^{13} \mathrm{C}$ NMR $\left(125.7 \mathrm{MH}_{\mathrm{Z}}, \mathrm{CDCl}_{3}\right): \delta=14.50$ and $19.27\left(2 \mathrm{CH}_{3}\right), 41.66$ and $41.97(2 \mathrm{CH}), 61.23$ $\left(\mathrm{CH}_{2}\right), 108.12(\mathrm{CN}), 114.62$ and $146.48(\mathrm{C}=\mathrm{C}), 128.25,128.95,129.45$, 136.47 (aromatic), 163.71 and $165.91(2 \mathrm{C}=\mathrm{O})$.

\section{Ethyl 5-cyano-2-methyl-6-oxo-4-phenyl-1,6-dihydropyridine 3-carboxylate (1B)}

White powder, $(59 \%)$, m.p. $143-145^{\circ} \mathrm{C}$, IR $(\mathrm{KBr})\left(v_{\max } / \mathrm{cm}^{-1}\right): 3411(\mathrm{NH}), 2234(\mathrm{CN})$, $1709(\mathrm{C}=\mathrm{O})$. MS, m/z (\%): $284\left(\mathrm{M}^{+}\right.$, 5). Anal. Calcd for $\mathrm{C}_{16} \mathrm{H}_{16} \mathrm{~N}_{2} \mathrm{O}_{3}: \mathrm{C}, 67.59 ; \mathrm{H}$, 5.67; N, $9.85 \%$. Found: $\mathrm{C}, 67.43 ; \mathrm{H}, 5.72 ; \mathrm{N}, 9.75 \% .{ }^{1} \mathrm{H}$ NMR $\left(500.1 \mathrm{MH}_{\mathrm{Z}}, \mathrm{CDCl}_{3}\right): \delta$ $=0.80\left(3 \mathrm{H}, \mathrm{t},{ }^{3} J_{\mathrm{HH}}=7 \mathrm{H}_{\mathrm{Z}}, \mathrm{CH}_{3}\right), 2.38\left(3 \mathrm{H}, \mathrm{s}, \mathrm{CH}_{3}\right), 4.09\left(2 \mathrm{H}, \mathrm{q},{ }^{3} J_{\mathrm{HH}}=7 \mathrm{H}_{\mathrm{Z}}, \mathrm{CH}_{2}\right)$, 7.26-7.61 (5H, m, aromatic), $13.38\left(1 \mathrm{H}\right.$, broad s, NH). ${ }^{13} \mathrm{C} \mathrm{NMR}\left(125.7 \mathrm{MH}_{\mathrm{Z}}, \mathrm{CDCl}_{3}\right): \delta$ $=14.50$ and $19.27\left(2 \mathrm{CH}_{3}\right), 41.66$ and $41.97(2 \mathrm{CH}), 61.23\left(\mathrm{CH}_{2}\right), 108.12(\mathrm{CN}), 114.62$ and $146.48(\mathrm{C}=\mathrm{C}), 128.25,128.95,129.45,136.47$ (aromatic), 163.71 and 165.91 $(2 \mathrm{C}=\mathrm{O})$.

Ethyl 5-cyano-2-methyl-6-oxo-4-(4-chlorophenyl)-1,6-dihydropyridine 3-carboxylate (2B)

White powder, (58 \%), m.p. $162-164^{\circ} \mathrm{C}$, IR $(\mathrm{KBr})\left(v_{\max } / \mathrm{cm}^{-1}\right): 3403(\mathrm{NH}), 2273(\mathrm{CN}), 1706$ $(\mathrm{C}=\mathrm{O})$. MS, $\mathrm{m} / \mathrm{z}(\%): 318\left(\mathrm{M}^{+}, 10\right)$. Anal. Calcd for $\mathrm{C}_{16} \mathrm{H}_{15} \mathrm{ClN}_{2} \mathrm{O}_{3}: \mathrm{C}, 60.29 ; \mathrm{H}, 4.74 ; \mathrm{N}$, 8.79\%. Found: $\mathrm{C}, 60.20 ; \mathrm{H}, 4.87 ; \mathrm{N}, 8.63 \%$. ${ }^{1} \mathrm{H}$ NMR $\left(500.1 \mathrm{MH}_{\mathrm{Z}}, \mathrm{CDCl}_{3}\right): \delta=1.21(3 \mathrm{H}, \mathrm{t}$, $\left.{ }^{3} J_{\mathrm{HH}}=7 \mathrm{H}_{\mathrm{Z}}, \mathrm{CH}_{3}\right), 2.49\left(3 \mathrm{H}, \mathrm{s}, \mathrm{CH}_{3}\right), 4.13\left(2 \mathrm{H}, \mathrm{q},{ }^{3} \mathrm{~J}_{\mathrm{HH}}=7 \mathrm{H}_{\mathrm{Z}}, \mathrm{CH}_{2}\right), 7.10-7.38(4 \mathrm{H}, \mathrm{m}$, aromatic), $12.74\left(1 \mathrm{H}\right.$, broad s, NH). ${ }^{13} \mathrm{C} \mathrm{NMR}\left(125.7 \mathrm{MH}_{\mathrm{Z}}, \mathrm{CDCl}_{3}\right): \delta=14.30$ and 18.51 $\left(2 \mathrm{CH}_{3}\right), 40,99$ and $41.83(2 \mathrm{CH}), 60.31\left(\mathrm{CH}_{2}\right), 104.62(\mathrm{CN}), 116.05$ and $148.30(\mathrm{C}=\mathrm{C})$, $129.17,130.04,132.99,137.50$ (aromatic), 163.28 and $166.10(2 \mathrm{C}=\mathrm{O})$. 
Ethyl 5-cyano-2-methyl-6-oxo-4-(4-bromophenyl)-1,4,5,6-tetrahydropyridine 3carboxylate (3A)

White powder, $(61 \%)$, m.p. $175-177^{\circ} \mathrm{C}$, IR $(\mathrm{KBr})\left(v_{\max } / \mathrm{cm}^{-1}\right): 3414(\mathrm{NH}), 2210(\mathrm{CN}), 1697$ $(\mathrm{C}=\mathrm{O})$. MS, $\mathrm{m} / \mathrm{z}(\%): 363\left(\mathrm{M}^{+}, 8\right)$. Anal. Calcd for $\mathrm{C}_{16} \mathrm{H}_{15} \mathrm{BrN}_{2} \mathrm{O}_{3}: \mathrm{C}, 52.91 ; \mathrm{H}, 4.16 ; \mathrm{N}$, 7.71\%. Found: C, 52.60; H, 4.25; N, 7.61\%. ${ }^{1} \mathrm{H}$ NMR $\left(500.1 \mathrm{MH}_{\mathrm{Z}}, \mathrm{CDCl}_{3}\right): \delta=1.22(3 \mathrm{H}, \mathrm{t}$, $\left.{ }^{3} J_{\mathrm{HH}}=7 \mathrm{H}_{\mathrm{Z}}, \mathrm{CH}_{3}\right), 2.46\left(3 \mathrm{H}, \mathrm{s}, \mathrm{CH}_{3}\right), 4.11\left(2 \mathrm{H}, \mathrm{q},{ }^{3} \mathrm{~J}_{\mathrm{HH}}=7 \mathrm{H}_{\mathrm{Z}}, \mathrm{CH}_{2}\right), 3.62\left(\mathrm{H}, \mathrm{d},{ }^{3} J_{\mathrm{HH}}=\right.$ $\left.7.1 \mathrm{H}_{\mathrm{Z}}, \mathrm{CH}\right), 4.47\left(\mathrm{H}, \mathrm{d},{ }^{3} J_{\mathrm{HH}}=7.1 \mathrm{H}_{\mathrm{Z}}, \mathrm{CH}\right), 7.12-7.49(4 \mathrm{H}, \mathrm{m}$, aromatic $), 7.84(1 \mathrm{H}$, broad s, $\mathrm{NH}) .{ }^{13} \mathrm{C} \mathrm{NMR}\left(125.7 \mathrm{MH}_{\mathrm{Z}}, \mathrm{CDCl}_{3}\right): \delta=14.12$ and $19.03\left(2 \mathrm{CH}_{3}\right), 40,99$ and $41.07(2 \mathrm{CH})$, $60.97\left(\mathrm{CH}_{2}\right), 107.38(\mathrm{CN}), 113.85$ and $146.05(\mathrm{C}=\mathrm{C}), 122.72,129.35,132.26,134.93$ (aromatic), 162.79 and $165.18(2 \mathrm{C}=\mathrm{O})$.

Ethyl 5-cyano-2-methyl-6-oxo-4-(4-bromophenyl)-1,6-dihydropyridine 3-carboxylate (3B)

White powder, $(61 \%)$, m.p. $175-177^{\circ} \mathrm{C}$, IR $(\mathrm{KBr})\left(v_{\max } / \mathrm{cm}^{-1}\right): 3414(\mathrm{NH}), 2210(\mathrm{CN}), 1697$ $(\mathrm{C}=\mathrm{O})$. MS, m/z (\%): $363\left(\mathrm{M}^{+}\right.$, 8). Anal. Calcd for $\mathrm{C}_{16} \mathrm{H}_{15} \mathrm{BrN}_{2} \mathrm{O}_{3}: \mathrm{C}, 52.91 ; \mathrm{H}, 4.16 ; \mathrm{N}$, 7.71\%. Found: C, 52.60; H, 4.25; N, 7.61\%. ${ }^{1} \mathrm{H}$ NMR (500.1 $\left.\mathrm{MH}_{\mathrm{Z}}, \mathrm{CDCl}_{3}\right): \delta=1.22(3 \mathrm{H}, \mathrm{t}$, $\left.{ }^{3} J_{\mathrm{HH}}=7 \mathrm{H}_{\mathrm{Z}}, \mathrm{CH}_{3}\right), 2.46\left(3 \mathrm{H}, \mathrm{s}, \mathrm{CH}_{3}\right), 4.11\left(2 \mathrm{H}, \mathrm{q},{ }^{3} J_{\mathrm{HH}}=7 \mathrm{H}_{\mathrm{Z}}, \mathrm{CH}_{2}\right), 7.12-7.49(4 \mathrm{H}, \mathrm{m}$, aromatic), 7.84(1 H, broad s, NH). $\left.{ }^{13} \mathrm{C} \mathrm{NMR} \mathrm{(125.7} \mathrm{MH}_{\mathrm{Z}}, \mathrm{CDCl}_{3}\right): \delta=14.12$ and 19.03 $\left(2 \mathrm{CH}_{3}\right), 40,99$ and $41.07(2 \mathrm{CH}), 60.97\left(\mathrm{CH}_{2}\right), 107.38(\mathrm{CN}), 113.85$ and $146.05(\mathrm{C}=\mathrm{C})$, $122.72,129.35,132.26,134.93$ (aromatic), 162.79 and $165.18(2 \mathrm{C}=\mathrm{O})$.

Ethyl5-cyano-2-methyl-6-oxo-4-(3-methoxyphenyl)-1,6-dihydropyridine3-carboxylate (4B)

White powder, $(57 \%)$, m.p. $134-136^{\circ} \mathrm{C}$, IR $(\mathrm{KBr})\left(v_{\max } / \mathrm{cm}^{-1}\right): 3415(\mathrm{NH}), 2220(\mathrm{CN}), 1705$ $(\mathrm{C}=\mathrm{O}) . \mathrm{MS}, \mathrm{m} / \mathrm{z}(\%): 314\left(\mathrm{M}^{+}\right.$, 12). Anal. Calcd for $\mathrm{C}_{17} \mathrm{H}_{18} \mathrm{~N}_{2} \mathrm{O}_{4}: \mathrm{C}, 64.96 ; \mathrm{H}, 5.77 ; \mathrm{N}, 8.91 \%$. Found: C, 64.88; H, 5.70; N, $8.95 \%$. ${ }^{1} \mathrm{H}$ NMR $\left(500.1 \mathrm{MH}_{\mathrm{Z}}, \mathrm{CDCl}_{3}\right): \delta=1.24\left(3 \mathrm{H}, \mathrm{t},{ }^{3} J_{\mathrm{HH}}=7 \mathrm{H}_{\mathrm{Z}}\right.$, $\left.\mathrm{CH}_{3}\right), 2.44\left(3 \mathrm{H}, \mathrm{s}, \mathrm{CH}_{3}\right), 3.80\left(3 \mathrm{H}, \mathrm{s}, \mathrm{OCH}_{3}\right), 4.16\left(2 \mathrm{H}, \mathrm{q},{ }^{3} J_{\mathrm{HH}}=7 \mathrm{H}_{\mathrm{Z}}, \mathrm{CH}_{2}\right), 6.79-7.29(4 \mathrm{H}, \mathrm{m}$, aromatic), $7.82(1 \mathrm{H}$, broad s, $\mathrm{NH}) .{ }^{13} \mathrm{C} \mathrm{NMR}\left(125.7 \mathrm{MH}_{\mathrm{Z}}, \mathrm{CDCl}_{3}\right): \delta=14.11$ and $18.87\left(2 \mathrm{CH}_{3}\right)$, 39.82 and $42.91(2 \mathrm{CH}), 55.23\left(\mathrm{OCH}_{3}\right), 60.82\left(\mathrm{CH}_{2}\right), 107.61(\mathrm{CN}), 113.39$ and $146.02(\mathrm{C}=\mathrm{C})$, 113.08, 114.13, 120.03, 130.07, 137.51159 .87 (aromatic), 163.20 and $165.02(2 \mathrm{C}=\mathrm{O})$.

Ethyl 5-cyano-2-methyl-6-oxo-4-(2-nitrophenyl)-1,6-dihydropyridine 3-carboxylate (5B)

White powder, $(54 \%)$, m.p. $166-168{ }^{\circ} \mathrm{C}$, IR $(\mathrm{KBr})\left(v_{\max } / \mathrm{cm}^{-1}\right): 3400(\mathrm{NH}), 2250(\mathrm{CN}), 1704$ $(\mathrm{C}=\mathrm{O}), 1346$ and $1523\left(\mathrm{NO}_{2}\right) .{ }^{1} \mathrm{H}$ NMR $\left(500.1 \mathrm{MH}_{\mathrm{Z}}, \mathrm{CDCl}_{3}\right): \delta=1.10\left(3 \mathrm{H}, \mathrm{t},{ }^{3} J_{\mathrm{HH}}=7 \mathrm{H}_{\mathrm{Z}}\right.$, $\left.\mathrm{CH}_{3}\right), 2.57\left(3 \mathrm{H}, \mathrm{s}, \mathrm{CH}_{3}\right), 4.04\left(2 \mathrm{H}, \mathrm{q},{ }^{3} J_{\mathrm{HH}}=7 \mathrm{H}_{\mathrm{Z}}, \mathrm{CH}_{2}\right), 7.20-7.98(4 \mathrm{H}, \mathrm{m}$, aromatic), 8.03(1 H, broad s, NH). ${ }^{13} \mathrm{C} \mathrm{NMR}\left(125.7 \mathrm{MH}_{\mathrm{Z}}, \mathrm{CDCl}_{3}\right): \delta=13.75$ and $18.91\left(2 \mathrm{CH}_{3}\right), 39.93$ and $41.95(2 \mathrm{CH}), 60.97\left(\mathrm{CH}_{2}\right), 106.66(\mathrm{CN}), 114.94$ and $147.46(\mathrm{C}=\mathrm{C}), 125.58,128.30$, $129.48,132.90,134.01,148.89$ (aromatic), 163.08 and $165.22(2 \mathrm{C}=\mathrm{O})$.

Ethyl 5-cyano-2-methyl-6-oxo-4-(3-nitrophenyl)-1,4,5,6-tetrahydropyridine 3carboxylate (6A)

White powder, $(68 \%)$, m.p. $190-192{ }^{\circ} \mathrm{C}$, IR $(\mathrm{KBr})\left(v_{\max } / \mathrm{cm}^{-1}\right): 3410(\mathrm{NH}), 2230(\mathrm{CN}), 1707$ $(\mathrm{C}=\mathrm{O}), 1319$ and $1525\left(\mathrm{NO}_{2}\right){ }_{1}^{1} \mathrm{H}$ NMR $\left(500.1 \mathrm{MH}_{\mathrm{Z}}, \mathrm{CDCl}_{3}\right): \delta=1.09\left(3 \mathrm{H}, \mathrm{t},{ }^{3} J_{\mathrm{HH}}=7 \mathrm{H}_{\mathrm{Z}}\right.$, $\left.\mathrm{CH}_{3}\right), 2.35\left(3 \mathrm{H}, \mathrm{s}, \mathrm{CH}_{3}\right), 4.01\left(2 \mathrm{H}, \mathrm{q},{ }^{3} J_{\mathrm{HH}}=7 \mathrm{H}_{\mathrm{Z}}, \mathrm{CH}_{2}\right), 4.31\left(\mathrm{H}, \mathrm{d},{ }^{3} J_{\mathrm{HH}}=7.1 \mathrm{H}_{\mathrm{Z}}, \mathrm{CH}\right)$, $4.47\left(\mathrm{H}, \mathrm{d},{ }^{3} \mathrm{~J}_{\mathrm{HH}}=7.1 \mathrm{H}_{\mathrm{Z}}, \mathrm{CH}\right), 7.46-8.09(4 \mathrm{H}, \mathrm{m}$, aromatic $), 10.54(1 \mathrm{H}$, broad s, NH$) .{ }^{13} \mathrm{C}$ NMR (125.7 $\left.\mathrm{MH}_{\mathrm{Z}}, \mathrm{CDCl}_{3}\right): \delta=14.04$ and $18.87\left(2 \mathrm{CH}_{3}\right), 44.81$ and $45.94(2 \mathrm{CH}), 65.28$ $\left(\mathrm{CH}_{2}\right), 110.05(\mathrm{CN}), 112.13$ and $153.14(\mathrm{C}=\mathrm{C}), 120.25,127.97,134.80,139.28,144.37$, 153.49 (aromatic), 163.97 and $166.92(2 \mathrm{C}=\mathrm{O})$. 
Ethyl 5-cyano-2-methyl-6-oxo-4-(3-nitrophenyl)-1,6-dihydropyridine 3-carboxylate (6B)

White powder, $\left(68 \%\right.$ ), m.p. $190-192{ }^{\circ} \mathrm{C}$, IR $(\mathrm{KBr})\left(v_{\max } / \mathrm{cm}^{-1}\right): 3410(\mathrm{NH}), 2230(\mathrm{CN}), 1707$ $(\mathrm{C}=\mathrm{O}), 1319$ and $1525\left(\mathrm{NO}_{2}\right){ }_{1}^{1} \mathrm{H}$ NMR $\left(500.1 \mathrm{MH}_{\mathrm{Z}}, \mathrm{CDCl}_{3}\right): \delta=1.09\left(3 \mathrm{H}, \mathrm{t},{ }^{3} J_{\mathrm{HH}}=7 \mathrm{H}_{\mathrm{Z}}\right.$, $\left.\mathrm{CH}_{3}\right), 2.35\left(3 \mathrm{H}, \mathrm{s}, \mathrm{CH}_{3}\right), 4.01\left(2 \mathrm{H}, \mathrm{q},{ }^{3} J_{\mathrm{HH}}=7 \mathrm{H}_{\mathrm{Z}}, \mathrm{CH}_{2}\right), 7.46-8.09$ (4H, m, aromatic), $10.54\left(1 \mathrm{H}\right.$, broad s, NH). ${ }^{13} \mathrm{C}$ NMR $\left(125.7 \mathrm{MH}_{\mathrm{Z}}, \mathrm{CDCl}_{3}\right): \delta=14.04$ and $18.87\left(2 \mathrm{CH}_{3}\right)$, 44.81 and $45.94(2 \mathrm{CH}), 65.28\left(\mathrm{CH}_{2}\right), 110.05(\mathrm{CN}), 112.13$ and $153.14(\mathrm{C}=\mathrm{C}), 120.25$, $127.97,134.80,139.28,144.37,153.49$ (aromatic), 163.97 and $166.92(2 \mathrm{C}=\mathrm{O})$.

\section{Results and Discussion}

Herein we report a new and efficient one-pot synthesis of polysubstituted dihydropyridone and pyridone derivatives by four-component reaction between cyanoacetamide, aryl aldehydes and ethyl acetoacetate with ammonium acetate in water. The reaction was performed in water under reflux conditions and afforded good yields of products. (Figure 1).

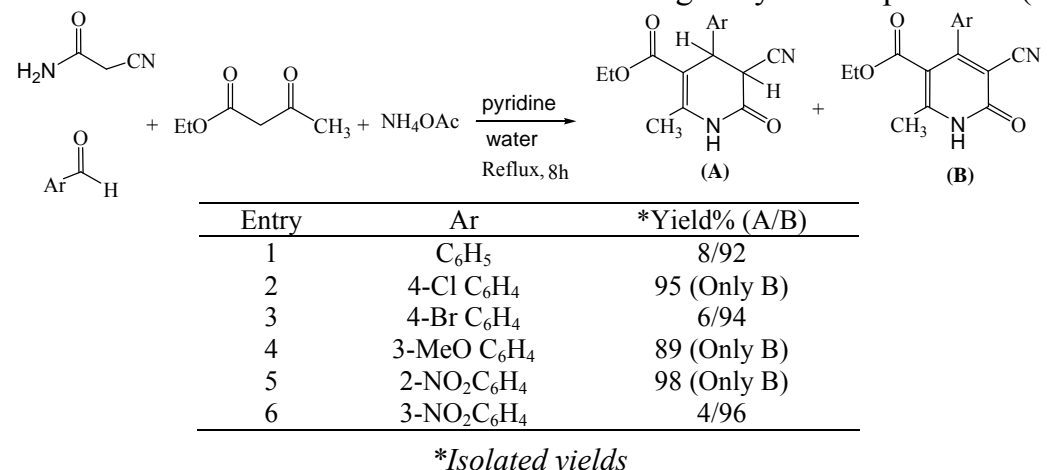

Figure 1. Four-component reaction between cyanoacetamide, aryl aldehydes and ethyl acetoacetate with ammonium acetate in water solvents.

The reaction course using water solvent afforded the product in higher yield and shorter reaction time. The structure of compounds $\mathbf{A}$ and $\mathbf{B}$ was deduced from their elemental analyses and their IR, ${ }^{1} \mathrm{H}$, and ${ }^{13} \mathrm{CNMR}$ spectral data. plausible mechanism may be advanced to rationalize product formation.

$$
\text { (x) }
$$

Figure 2. Suggested mechanism for formation of compound $\mathbf{A}$ and $\mathbf{B}$ 
Resumably a intermediate $\mathbf{3}$ formed from Michael addition of product $\mathbf{1}$ the addition of cyanoacetamide with aryl aldehydes and product 2 the addition of ethyl acetoacetate with ammonium acetate which could undergo stepwise cyclization to produce $\mathbf{A}$ by elimination of $\mathrm{NH}_{3}$. The amide tautomer is considerably more stable. Admittedly the exchangeable peak in the $1 \mathrm{H}$ NMR spectrum is very high for a NH peak, and would appear to be more consistent with a $\mathrm{OH}$ peak. The $\mathbf{A}$ product is finally converted to $\mathbf{B}$ by oxidation (Figure 2).

\section{Conclusion}

We have reported a four-component reaction between cyanoacetamide, aryl aldehydes and ethyl acetoacetate with ammonium acetate. The reaction was performed in water under reflux conditions and afforded good yields of products. The present method carries the advantage that not only is the reaction performed under neutral conditions but also that the substances can be mixed without any activation or modification.

\section{Acknowledgement}

We gratefully acknowledge financial support from the Research Council of Islamic Azad University, Yazd branch.

\section{References}

1. Misic-Vukovic M, Mijin D, Radojkovic-Velickovic M, Valentic N and Krstic V, $J$ Serb Chem Soc., 1998, 63, 585.

2. Balasubramanian M, Keay J G, Katritzky A R, Rees C W and Scriven E V F, Comprehensive Heterocyclic Chemistry II Vol. 5, Pergamon Press, London, 1996, pp. 245-300 Chapter 6.

3. Constable E C, Housecroft C E, Neuburger M, Phillips D, Raithby P R, Schofield E, Sparr E, Tocher D A, Zehnder M and Zimmermann Y, J Chem. Soc Dalton Trans.. 2000, 13, 2219-2228; DOI:10.1039/B000940G

4. Kim B Y, Ahn J B, Lee H W, Kang S K, Lee J H, Shin J S, Ahn S K, Hong C I and Yoon S S, Eur J Med Chem., 2004, 39(5), 433-447;DOI:10.1016/j.ejmech.2004.03.001

5. Enyedy I J, Sakamuri S, Zaman W A, Johnson K M and Wang S, Bioorg Med Chem Lett., 2003, 13(3), 513-517; DOI:10.1016/S0960-894X(02)00943-5

6. Pillai A D, Rathod P D, Franklin P X, Patel M, Nivsarkar M, Vasu K K, Padh H and Sudarsanam V, Biochem Biophys Res Commun., 2003, 301(1), 183-196; DOI:10.1016/S0006-291X(02)02996-0

7. Klimešová V, Svoboda M, Waisser K, Pour M and Kaustová J, II Farmaco, 1999, 54(10), 666-672; DOI:10.1016/S0014-827X(99)00078-6

8. Jones G, Katritzky A R, Rees C W and Scriven E V F, Editors, Comprehensive Heterocyclic Chemistry II Vol. 5, Pergamon Press, London, 1996, pp. 168-243 Chapter 5 and references cited therein.

9. Katritzky A R, Abdel-Fattah A A A, Tymoshenko D O and Essawy S A, Synthesis, 1999, 12, 2114.

10. Rong L, Han H X, Jiang H, Zhang Q and Tu S, Synth Commun., 2009, 39(6), $1027-$ 1034; DOI:10.1080/00397910802463878

11. Khazaei M and Anary-Abbasinejad M, Chem Sci Trans., 2014, 3(3), 977-982; DOI:10.7598/cst2014.204

12. Khazaei M, Anary-Abbasinejad M, Hassanabadi A and Sadeghi B, J Chem., 2012, 9(2), 615-620. 